\title{
Development and Evaluation of the Behavioral and Psychological Symptoms of Dementia Questionnaire 13-Item Version (BPSD13Q)
}

\author{
Taiga Fuju ${ }^{a, b}$ Tetsuya Yamagami ${ }^{c}$ Mio Ito $^{c}$ Noriko Naito $^{d}$ \\ Haruyasu Yamaguchi ${ }^{\mathrm{a}}$ \\ aTokyo Center for Dementia Care Research and Practices, Suginami, Japan; ${ }^{\text {b}}$ Medical Corporation TAISEIKAI/UCHIDA \\ Hospital, Numata, Japan; 'Graduate School of Health Sciences, Gunma University, Maebashi, Japan; ' Yokufukai \\ Hospital, Suginami, Japan
}

\section{Keywords}

Behavioral and psychological symptoms of dementia .

Scale · Dementia - Dementia care - Behavioral and psychological symptoms of sementia questionnaire 13-item version

\begin{abstract}
Introduction: Most behavioral and psychological symptoms of dementia (BPSD) scales have copyright issues and are difficult for care staff to use in daily care settings because they were primarily designed for physicians. Therefore, an easier tool for care staff is required. This study aimed to develop and validate the BPSD questionnaire 13-item version (BPSD13Q). Methods: We obtained data from 444 people with dementia living in group homes in Japan using the BPSD plus questionnaire (BPSD + Q; 27-item version) and Neuropsychiatric Inventory Nursing Home version (NPI-NH). We selected appropriate items to make a short-form version of the $\mathrm{BPSD}+\mathrm{Q}$ and examined the construct validity, internal consistency, and criterion-related validity of the questionnaire. Results: By the pilot review, research on correlations with similar items from comparable scales, and factor analysis, we reduced 27 items to 13 items (BPSD13Q). The BPSD13Q and BPSD13Q-distress (BPSD13Q-D) showed good internal consistency (Cronbach's $\alpha=0.76$ and 0.80 , respectively). More-
\end{abstract}

karger@karger.com www.karger.com/dee

Karger $\stackrel{\text { ' }}{5}$

GOPEN ACCESS
C 2021 The Author(s).

Published by S. Karger AG, Basel

This is an Open Access article licensed under the Creative Commons Attribution-NonCommercial-4.0 International License (CC BY-NC) (http://www.karger.com/Services/OpenAccessLicense), applicable to the online version of the article only. Usage and distribution for commercial purposes requires written permission. over, the BPSD13Q was positively correlated with the NPI-NH $(r=0.72, p<0.001)$ and BPSD + Q $(r=0.95, p<0.001)$. The BPSD13Q-D was positively correlated with the NPI-NH-caregiver distress $(r=0.74, p<0.001)$ and BPSD + Q-distress $(r=$ $0.96, p<0.001)$. Conclusion: We developed and validated the BPSD13Q, which is a short-form version of the BPSD + Q and is downloadable. The BPSD13Q may make BPSD evaluations easier for the care staff.

(c) 2021 The Author(s).

Published by S. Karger AG, Basel

\section{Introduction}

The number of people with dementia is increasing globally [1]. The antecedents and triggers of behavioral and psychological symptoms of dementia (BPSD) have been focused upon for decades to reduce BPSD [2], and initiatives to prevent BPSD have been promoted in Japan [3]. We consider prevention of BPSD as a new and important concept because BPSD causes a great amount of stress for people with dementia as well as care burdens for caregivers [4]. Although it is necessary to evaluate and analyze the effects of dementia care for preventing BPSD, the ordinary scales such as the Neuropsychiatric Inventory (NPI) [5] and Dementia Behavior Disturbance (DBD) scale [6] have some problems concerning usage. 
The NPI Nursing Home version (NPI-NH) [7] and NPI Questionnaire (NPI-Q) [8] are copyright-protected. Moreover, the NPI and the NPI-NH are difficult for care staff to use because of the interview format. Additionally, the DBD scale consists of only behavioral symptoms and not psychological symptoms. Hence, to the best of our knowledge, there is no assessment scale for BPSD that is useful to on-site care staff.

The BPSD plus questionnaire (BPSD + Q) consists of 27 items, 25 of which are related to BPSD (BPSD questionnaire 25-item version; BPSD25Q) and 2 are related to delirium [9]. The 25 items of BPSD were created based on written diagnoses from primary physicians for long-term care insurance and interviews with the care staff. Moreover, the BPSD + Q consists of 3 subscales: hyperactive (13 items), hypoactive (6 items), and daily living-related symptoms (6 items). Thus, the BPSD + Q evaluates BPSD precisely, but some items that hardly emerge in care facilities are included. Furthermore, it takes approximately 7-9 min to complete the questionnaire, which places a burden on the care staff [10]. Although we recommend using the BPSD + Q for evaluating various kinds of BPSD in detail, the development of a short version is expected for the care staff. Therefore, we developed a BPSD assessment scale, the BPSD questionnaire 13-item version (BPSD13Q), a 13-item short version of the BPSD + Q.

\section{Materials and Methods}

\section{Participants}

The participants were 444 people with dementia living in group homes in Japan, and we analyzed the data of their NPI-NH and BPSD $+\mathrm{Q}$ scores $[10,11]$. The mean age of the participants was $87.3 \pm 7.3$ years, and 364 were females. Of the 444 participants, 296 had Alzheimer's disease, 39 vascular dementia, 21 Lewy body dementia, 13 mixed-type dementia, 7 frontotemporal dementia, 8 did not clarify, and 60 selected "none of the above."

For conducting a frequency calculation of BPSD, we used published data from our studies of Naito et al. [9] and Fuju et al. [10]. In addition to the 444 participants from these studies $[10,11]$, the data of 83 (mean age $83.2 \pm 7.1$ years; 57 females; 58 with Alzheimer's disease) and 130 people with dementia (mean age $85.9 \pm 7.5$ years; 107 females, 50 with Alzheimer's disease) from the studies of Naito et al. [9] and Fuju et al. [10], respectively, were analyzed.

\section{$B P S D+Q$ and NPI-NH}

The severity of each item in the BPSD + Q is assessed on a scale ranging from 0 to 5 . Higher scores indicate a greater severity of BPSD. The BPSD + Q-distress (BPSD + Q-D) evaluates the degree of caregiver burden for each item, rated on a scale ranging from 0 to 5. Higher scores indicate a higher degree of caregiver burden.

The NPI-NH [7] consists of 12 BPSD-related items (delusions, hallucinations, agitation/aggression, dysphoria/depression, anxi- ety, euphoria/elation, apathy/indifference, disinhibition, irritability/liability, aberrant motor behavior, nighttime behavioral disturbances, and appetite/eating disturbances). Each item is assessed by frequency (range $=0-4) \times$ severity (range $=1-3$ ) based on interviews. The total scores are calculated based on 10 items, excluding nighttime and appetite/eating disturbances (range $=0-120)$. Higher scores indicate greater severity of BPSD. The NPI Caregiver Distress Scale (NPI-D) evaluates the degree of caregiver burden for each NPI-NH item, rated on a scale ranging from 0 to 5 , with total scores ranging from 0 to 50 . Higher scores indicate higher caregiver burden. The scale of BPSD was assessed by care staff.

\section{Low-Frequency Item Reduction from BPSD $+Q$}

We calculated the mean frequency of each of the 25 items (excluding the 2 items related to delirium) based on previous studies $[9,10]$ and eliminated the items with low frequency (BPSD frequency $\leq 20 \%$ ) through discussion with a physician who specializes in dementia, 2 physical therapists, and a nurse.

\section{Item Reduction from the Construct Validation Study and}

\section{Collateral Validation Study}

We calculated correlations between items of BPSD severity and eliminated items with high correlation levels $(\rho>0.5)$. The factor structure was determined by factor analysis with an oblique promax rotation. We retained the factors with an eigenvalue $>1$. The threshold level for factor loading was 0.30 . The data were used by BPSD severity.

\section{Reliability (Internal Consistency) and Validity}

(Criterion-Related Validity) of the Short Version

We evaluated internal consistency using Cronbach's a and accepted the items that obtained a score of 0.7 or higher. The validity of the BPSD13Q was analyzed using the Spearman's correlation coefficients between the BPSD13Q and the NPI-NH.

\section{Results}

\section{Low-Frequency Item Reduction}

First, we decided to delete "hyperactive delirium" and "hypoactive delirium" because they did not pertain to BPSD. Second, we calculated the mean frequency of BPSD in the review $[9,10]$, and 9 items showed a mean frequency of BPSD <20\%: physically aggressive behavior, going out without telling anybody, inappropriate sexual behavior, inappropriate vocalization, withdrawal, changes in eating behaviors, carelessness of fire, uncleanliness, and hiding and losing things. We reduced these 9 items.

\section{Item Reduction from the Construct Validation Study} and Collateral Validation Study

"Irritability" and "apathy" showed a significant and positive correlation with "verbally aggressive behavior" $(\rho=0.58)$ and "lack of interest" $(\rho=0.59)$, respectively; therefore, we deleted "verbally aggressive behavior" and 
Table 1. Component factors of the BPSD13Q

\begin{tabular}{llllll}
\hline Item & \multicolumn{7}{l}{ Factor loading } & & & \\
\cline { 2 - 6 } & I & II & III & IV & V \\
\hline Anxiety & 1.035 & 0.025 & -0.027 & 0.024 & -0.115 \\
Depression & 0.424 & -0.093 & -0.010 & 0.344 & 0.229 \\
Repeated questions & 0.397 & 0.099 & 0.232 & -0.144 & 0.041 \\
Irritability & 0.014 & 0.968 & -0.118 & -0.053 & 0.155 \\
Resistance to care & -0.030 & 0.529 & 0.105 & 0.230 & -0.172 \\
Disinhibition & 0.089 & 0.393 & 0.048 & -0.065 & 0.195 \\
Wandering and restlessness & 0.072 & 0.019 & 0.879 & -0.127 & -0.061 \\
Day-night reversal & -0.063 & -0.027 & 0.508 & 0.170 & 0.065 \\
Stereotyped behavior & 0.052 & 0.023 & 0.366 & 0.019 & 0.079 \\
Apathy & 0.009 & 0.027 & -0.053 & 0.763 & 0.033 \\
Somnolence tendency & 0.007 & 0.079 & 0.162 & 0.577 & -0.070 \\
Delusion & -0.022 & 0.110 & 0.033 & -0.055 & 0.654 \\
Visual and auditory hallucinations & 0.025 & 0.012 & 0.202 & 0.138 & 0.336 \\
\hline
\end{tabular}

Factor analysis was conducted with the oblique promax rotation. Bartlett's test of sphericity was significant at $<0.01$, and the Kaiser-Meyer-Olkin value was 0.71. The BPSD13Q obtained Cronbach's a value of 0.76. BPSD13Q, behavioral and psychological symptoms of dementia questionnaire 13-item version.

Fig. 1. Correlation between the BPSD13Q scores and the scores of the NPI-NH (a) and BPSD + Q (b). a The BPSD13Q scores show significant and positive correlations with the NPI-NH scores $(r=0.72, p<$ 0.001). b The BPSD13Q scores show significant and positive correlations with the BPSD + Q scores $(r=0.95, p<0.001)$. BPSD13Q, behavioral and psychological symptoms of dementia questionnaire 13-item version; NPI-NH, Neuropsychiatric Inventory-Nursing Home version; BPSD + $\mathrm{Q}$, behavioral and psychological symptoms of dementia plus questionnaire.

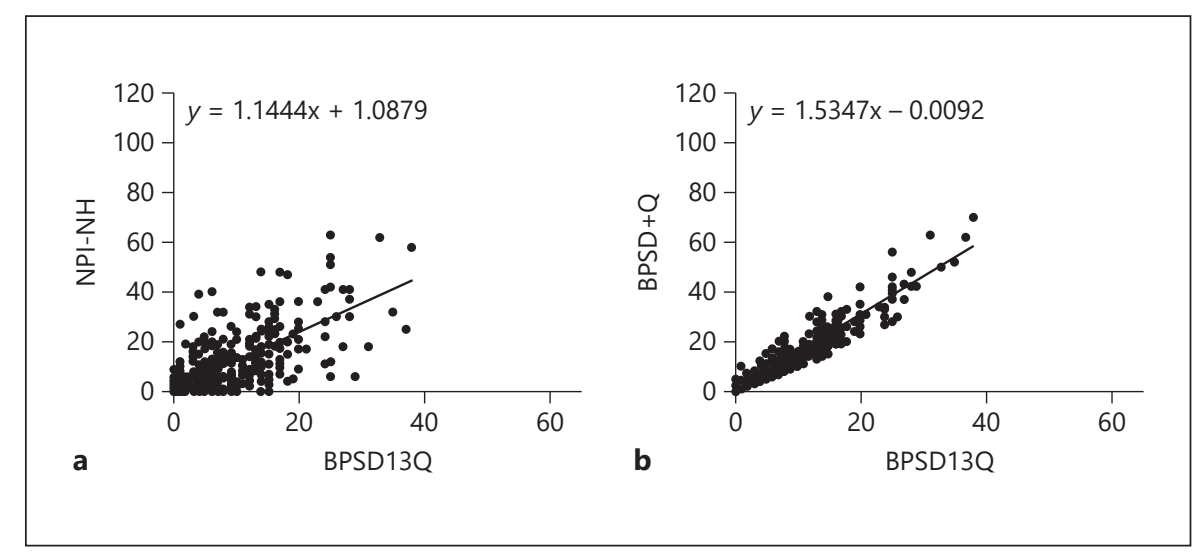

"lack of interest" to avoid redundancy. We reduced the 16 items to 14 items. Finally, 3-factor analyses were conducted. After the first factor analysis with 14 items was conducted, we decided to exclude the "hoarding behavior" as it showed factor loadings of $<0.3$. In the second factor analysis, a 5-factor structure from the Kaiser-Guttman rule was assumed. In the third factor analysis, all 13 items showed sufficient factor loadings. Table 1 lists the items and their factor loadings. Bartlett's test of sphericity was significant at more than 0.01, and the Kaiser-MeyerOlkin value was 0.71 , confirming that the data were suitable for factor analysis.
Reliability (Internal Consistency) and Validity

(Criterion-Related Validity) of the BPSD13Q

Through the aforementioned analysis, we fixed 13 items (BPSD13Q). Cronbach's a of the whole severity of 13 items was 0.76 , and the caregiver burden (distress) for each item was 0.80 .

The BPSD13Q total scores were significantly and positively correlated with the NPI-NH total scores $(r=0.72$, $p<0.001$; Fig. 1a), BPSD25Q $(r=0.96, p<0.001)$, and $\mathrm{BPSD}+\mathrm{Q}(r=0.95, p<0.001$; Fig. 1b). The total scores of the BPSD13Q-distress (BPSD13Q-D) were significantly and positively correlated with the NPI-D $(r=0.74, p<$ 0.001; Fig. 2a), BPSD25Q-D $(r=0.96, p<0.001)$, and BPSD + Q-D ( $r=0.96, p<0.001$; Fig. $2 \mathrm{~b})$. 
Fig. 2. Correlation between the BPSD13Q$\mathrm{D}$ scores and the scores of the NPI-D (a) and BPSD + Q-D (b). a The BPSD13Q-D scores show significant and positive correlations with the NPI-D scores $(r=0.74, p<$ 0.001). b The BPSD13Q-D scores show significant and positive correlations with the BPSD + Q-D scores $(r=0.96, p<0.001)$. BPSD13Q-D, behavioral and psychological symptoms of dementia questionnaire 13item version-distress; NPI-D, Neuropsychiatric Inventory Caregiver Distress Scale; BPSD + Q-D, behavioral and psychological symptoms of dementia plus questionnairedistress.

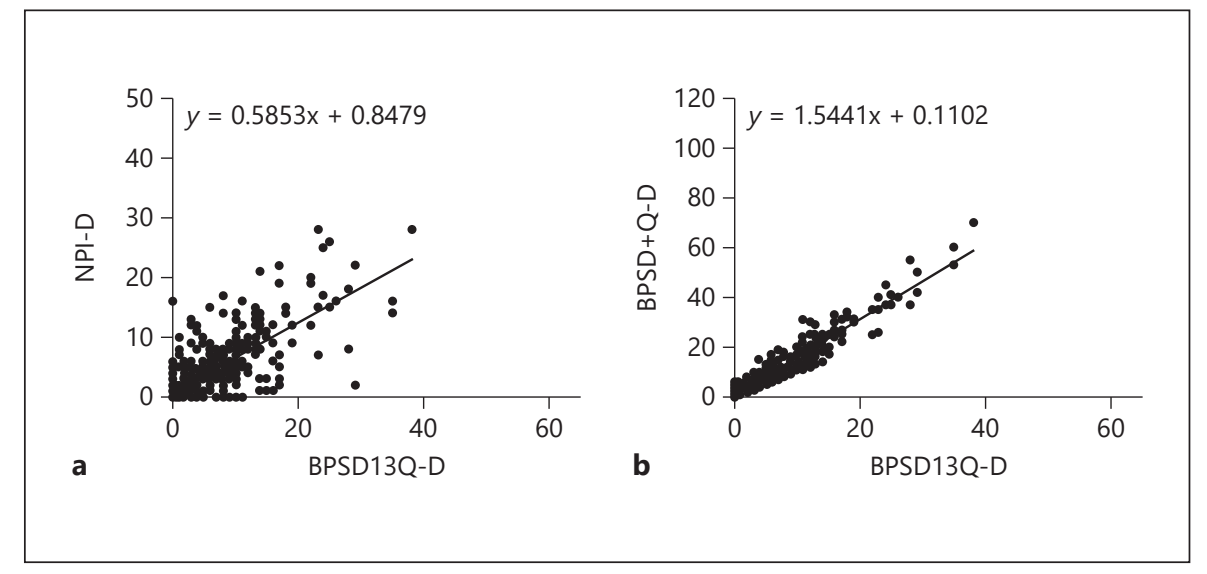

Moreover, the total scores of the BPSD13Q showed significant and positive correlations with the scores of the BPSD + Q subscale "hyperactive symptoms" $(r=0.83$, $p<0.001)$, "hypoactive symptoms" $(r=0.70, p<0.001)$, and "daily living-related symptoms" $(r=0.68, p<0.001)$. The total scores of the BPSD13Q-D showed significant and positive correlations with the scores of the BPSD + Q-D subscale "hyperactive symptoms" $(r=0.87, p<$ $0.001)$, "hypoactive symptoms" $(r=0.69, p<0.001)$, and "daily living-related symptoms" $(r=0.73, p<0.001)$.

\section{Discussion/Conclusion}

We developed the BPSD13Q, a short version of the $\mathrm{BPSD}+\mathrm{Q}$, and proved its validity and reliability. The scores of the BPSD13Q showed sufficient correlations with the NPI-NH and the BPSD + Q scores, and the same analysis was conducted for the BPSD13Q-D.

The items of the BPSD13Q were categorized into 5 factors, similar to those shown in previous studies $[12,13]$. "Depression" and "anxiety" are usually found in the same factor in most studies [12-16], and according to Phannarus et al. [15], "repeating" is also included in the same factor [15]. The second factor included "irritability," "resistance to care," and "disinhibition." Therefore, it is assumed that "irritability" and "disinhibition" are included in the same factor $[12,13]$, and "resistance to care" is included in the same factor as hyperactive-type behavioral symptoms $[12,13,15,16]$. "Wondering and restlessness," "day-night reversal," and "stereotyped behavior" were categorized chiefly into the agitated behavioral symptoms. "Apathy" and "somnolence tendency" were categorized as hypoactive symptoms. "Delusion" and "visual and auditory hallucinations" usually co-occur $[12,13,16$, 17]. In this way, our results were similar to those of previous studies and are therefore structurally valid. Recently, the 14-item Thai language BPSD scoring system for dementia (BPSD-T) has been developed for nonphysician health-care personnel [15], but our evaluation scale has the advantage of being easy to use even for care staff who are often involved in the actual dementia care.

A limitation of this study is that intra- and inter-rater reliabilities were not examined, and further consideration is needed. The BPSD13Q included most of the items of the NPI-NH [7], evaluated both severity and frequency, and showed good internal consistency and construct validity. Furthermore, both English and Japanese versions of the BPSD13Q are downloadable at DCnet (https://www.dcnet.gr.jp/support/evaluation/index. php). Therefore, it is considered that the BPSD13Q can be useful for easy assessment of BPSD. It is advised to use the BPSD + Q for evaluating a variety of BPSD in detail and use the BPSD13Q for easy on-site evaluation of major BPSD.

Care for BPSD is generally administered by care staff. It is expected that the BPSD13Q will help care staff evaluate BPSD in a short time and contribute to more effective care. We hope that the BPSD13Q will contribute to the development of effective care skills to prevent BPSD as prevention is much more important than care after its outbreak [4].

\section{Acknowledgement}

The authors thank all the study participants, the study supporters, and the Japan Group-Home Association for People with Dementia. Special thanks also go to Hiroki Kawashima for his advice 
on translation from Japanese to English. This study was written as one of the research results of Japan Agency for Medical Research and Development (AMED) (Grant No. JP20us0424001).

\section{Statement of Ethics}

The subjects provided informed consent, and the study protocol was approved by the Ethics Committees of the Japan GroupHome Association for People with Dementia (Approval No: 2) and the Tokyo Center for Dementia Care Research and Practices (Approval No: 15, 20). Moreover, the subjects provided informed consent for the secondary use of the anonymized data. The study was conducted in accordance with the ethical standards of the Declaration of Helsinki, and written informed consent was obtained from all participants.

\section{Conflict of Interest Statement}

The authors have no potential conflicts of interest to disclose.

\section{Funding Sources}

This study used data from the Grant from the Health and Welfare Bureau for the Elderly (Ministry of Health, Labour, and Welfare) [11]. This research was supported by AMED under Grant Number JP20us0424001.

\section{Author Contributions}

T. Fuju conducted the study, performed the statistical analyses, interpreted the data, and wrote the manuscript. T. Yamagami, M. Ito, and N. Naito conducted the study and wrote and revised the manuscript. H. Yamaguchi conceived the study, advised statistical analyses, and critically revised the manuscript. All authors have read and approved the final manuscript.

\section{Data Availability Statement}

Publicly available datasets were used in this study. These can be found in the references [9-11].

\section{References}

1 Prince M, Wimo A, Guerchet M, Ali GC, Wu YT, Prina M. World Alzheimer Report 2015. The global impact of dementia. An analysis of prevalence, Alzheimer's incidence, cost and trends. London: Alzheimer's Disease International; 2015.

2 Smith M, Gerdner LA, Hall GR, Buckwalter KC. History, development, and future of the progressively lowered stress threshold: a conceptual model for dementia care. J Am Geriatr Soc. 2004 Oct;52(10):1755-60.

3 Ministry of Health, Labour and Welfare of Japan. Japanese Dementia Strategy. [Internet]; 2019. [cited $2020 \mathrm{Jul}$ 17]. Available from: https://www.mhlw.go.jp/content/000522832. pdf. Japanese.

4 Yamaguchi H, Fuju T. Change from "classification" to "perspective" for symptoms of dementia: focused on BPSD. Dementia Japan. 2021 April;35(2):226-40. Japanese.

5 Cummings JL. The Neuropsychiatric Inventory: assessing psychopathology in dementia patients. Neurology. 1997 May;48(5 Suppl 6): S10-6.

6 Baumgarten M, Becker R, Gauthier S. Validity and reliability of the dementia behavior disturbance scale. J Am Geriatr Soc. 1990 Mar;38(3):221-6.

7 Wood S, Cummings JL, Hsu MA, Barclay T, Wheatley MV, Yarema KT, et al. The use of the neuropsychiatric inventory in nursing home residents. Characterization and measurement. Am J Geriatr Psychiatry. 2000;8(1): 75-83.
8 Kaufer DI, Cummings JL, Ketchel P, Smith V, MacMillan A, Shelley T, et al. Validation of the NPI-Q, a brief clinical form of the Neuropsychiatric Inventory. J Neuropsychiatry Clin Neurosci. 2000 Spring; 12(2):233-9.

9 Naito N, Fuju T, Takiguchi Y, Ito M, Yamagami T, Yamaguchi $\mathrm{H}$. Development and evaluation of a new BPSD questionnaire, BPSD+Q. Tokyo J Dementia Care Res. 2018 Dec;2:133-45. Japanese.

10 Fuju T, Naito N, Takiguchi Y, Nakamura K, Yamaguchi $\mathrm{H}$. A comparative study on the usefulness of the BPSD + Q with that of the NPI-Q and the NPI-NH based on primary doctor judgment for long-term care insurance in care facilities. Jap J Geriatr Psychiatry. 2020 Apr;31(4):389-402. Japanese.

11 Japan Group-Home Association for people with Dementia. Report funded by the Health and Welfare Bureau for the Elderly (Ministry of Health, Labour, and Welfare) [Internet]. [cited 2021 June 17]. Available from: https:// www.ghkyo.or.jp/news/wp-content/uploads/2019/04/310410_H30Reasearch.pdf. Japanese.

12 Aalten P, Verhey FR, Boziki M, Bullock R, Byrne EJ, Camus V, et al. Neuropsychiatric syndromes in dementia. Results from the European Alzheimer Disease Consortium: part I. Dement Geriatr Cogn Disord. 2007 Nov; 24(6):457-63.
13 Aalten P, Verhey FR, Boziki M, Brugnolo A Bullock R, Byrne EJ, et al. Consistency of neuropsychiatric syndromes across dementias: results from the European Alzheimer Disease Consortium. Part II. Dement Geriatr Cogn Disord. 2008 Nov;25(1):1-8.

14 Vaingankar JA, Chong SA, Abdin E, Picco L, Jeyagurunathan A, Seow E, et al. Behavioral and psychological symptoms of dementia: prevalence, symptom groups and their correlates in community-based older adults with dementia in Singapore. Int Psychogeriatr. 2017 Aug;29(8):1363-76.

15 Phannarus H, Muangpaisan W, Siritipakorn P, Pianchob S, Supapueng O. Development of a Thai tool for assessing behavioral and psychological symptoms of dementia: a confirmatory factor analysis. Brain Behav. 2020 Nov;10(11):e01816.

16 Feghali Y, Fares Y, Abou Abbas L. Assessment of neuropsychiatric symptoms in dementia: validity and reliability of the Lebanese version of the neuropsychiatric inventory questionnaire. Appl Neuropsychol Adult. 2019 Sep;26: $1-8$

17 Makimoto K, Kang Y, Kobayashi S, Liao XY, Panuthai S, Sung HC, et al. Prevalence of behavioural and psychological symptoms of dementia in cognitively impaired elderly residents of long-term care facilities in East Asia: a cross-sectional study. Psychogeriatrics. 2019 Mar;19(2):171-80. 